\title{
Factors Associated with Pain Treatment Satisfaction Among Patients with Chronic Non-Cancer Pain and Substance Use
}

\author{
Leslie W. Suen, MD, Vanessa M. McMahan, PhD, Christopher Rowe, PhD, \\ Sumeet Bhardwaj, MD, Kelly Knight, PhD, Margot B. Kushel, MD, \\ Glenn-Milo Santos, PhD, and Phillip Coffin, MD
}

Introduction: A better understanding of pain treatment satisfaction in patients with chronic noncancer pain (CNCP) and substance use is needed, especially as opioid prescribing policies are changing. We sought to identify factors associated with pain treatment satisfaction in individuals with CNCP on recent opioid therapy and prior or active substance use.

Methods: An exploratory cross-sectional analysis using baseline data from a cohort study of 300 adults with CNCP receiving $>20$ morphine milligram equivalents of opioids for $\geq 3$ of the preceding 12 months and prior or active substance use. Participants completed interviews, clinical assessments, urine drug screening, and medical chart review.

Results: Participants were predominantly middle-aged (mean age 57.5 years), Black (44\%), and cisgender men (60\%). One-third (33\%) had high, $28 \%$ moderate, and 39\% low pain treatment satisfaction. Post-traumatic stress disorder (PTSD), tobacco use, past-year opioid discontinuation, and higher average pain scores were associated with lower satisfaction. HIV and prescription cannabis use were associated with higher satisfaction.

Conclusions: The relationship between PTSD and tobacco use with lower satisfaction should be explored to augment pain outcomes. Higher satisfaction among individuals with HIV and prescription cannabis use presents potential research areas to guide CNCP management and reduce reliance on opioid therapies. (J Am Board Fam Med 2021;34:1082-1095.)

Keywords: Chronic Disease, Chronic Pain, Cross-Sectional Studies, Opioids, Patient Satisfaction, Policy, Primary Health Care, Prospective Studies

\section{Introduction}

One in 5 adults in the US experiences chronic noncancer pain (CNCP), or noncancer pain persisting for at least 3 months. ${ }^{1}$ It is one of the most common reasons for seeking primary care. ${ }^{1,2} \mathrm{CNCP}$ is challenging

This article was externally peer reviewed.

Submitted 20 May 2021; revised 9 July 2021; accepted 26 July 2021.

From National Clinician Scholars Program, Philip R. Lee Institute of Health Policy Studies, University of California San Francisco, San Francisco, CA (LWS); San Francisco Veterans Affairs Medical Center, San Francisco, CA (LWS); San Francisco Department of Public Health, San Francisco, CA (VMM, CR, GS, PC); University of Toronto, Department of Family and Community Medicine, Toronto, ON, Canada (SB); Department of Humanities and Social Sciences, University of California San Francisco, San Francisco, CA (KK); Center for Vulnerable Populations, University of California San Francisco, San Francisco, CA (MBK); Department of Community Health Systems, University of California, San Francisco, San Francisco, CA for primary care providers (PCPs), due to a lack of proven treatments, heterogeneity of pain conditions, and limited duration of treatment studies. ${ }^{2,3}$ In safetynet settings, CNCP patients face a higher prevalence of comorbid conditions associated with worse pain

(GS); Department of Medicine, University of California, San Francisco, San Francisco, CA (PC).

Funding: This work was supported by the National Institute on Drug Abuse (R01DA040189 and K24DA042720) and the National Heart, Lung and Blood Institute (R38HL143581). The funding agency had no role in study design, data collection, analysis, the decision to publish, or the preparation of the manuscript

Conflicts of interest: MBK serves in a leadership role in Housing California. The authors declare no other potential conflicts of interest.

Corresponding author: Leslie Suen, MD, UCSF National Clinician Scholars Program, Philip R. Lee Institute of Health Policy Studies, 490 Illinois St., Suite 7227, Box 0936, San Francisco, CA 94158 (E-mail: Leslie.Suen@ucsf.edu). 
outcomes, including substance use disorders, ${ }^{4-6}$ as well as barriers to accessing multimodal pain therapies and pain management resources. $5,7,8$

Among patients with $\mathrm{CNCP}$, pain treatment satisfaction is a common indicator for quality of care for public health systems and can affect patient adherence to treatment. ${ }^{9-11}$ Pain treatment satisfaction can be affected by multiple factors, including patient views of care providers, experiences and expectations with pain management, and pain outcomes. ${ }^{12}$ Satisfaction is higher when patients are treated as informed partners in their pain treatment plans. ${ }^{12,13}$ CNCP patients who use substances also usually have lower pain treatment satisfaction, potentially due to stigmatization in health settings, leading to strained relationships with providers. $5,9,14$ In safety-net populations, patients dissatisfied with their pain treatment have lower care retention, lower adherence and engagement with treatment plans, and worse health outcomes. ${ }^{9,11}$

Opioids were previously considered a mainstay of CNCP treatment. ${ }^{15,16}$ However, implementation of opioid-limiting policies has led prescriptions to decline nationally since $2012 .^{17,18}$ The national rate of high-dosage opioid prescriptions declined by 4 prescriptions per month from 2012 to 2016, and this decrease doubled after release of the Centers for Disease Control and Prevention (CDC) Guideline for Prescribing Opioids for Chronic Pain. ${ }^{18}$ Changes in prescribing policies are salient in safety-net settings, where the high prevalence of co-occurring substance use raises safety concerns of increased overdose risk for patients on opioid therapy. ${ }^{8,15,19,20}$ In 2016, the San Francisco Health Network (SFHN), the main primary care health system serving publicly insured and uninsured patients in San Francisco, implemented several county-wide policies to limit opioid prescribing. ${ }^{21,22}$ From 2016 to 2019, the number of opioid prescriptions issued in San Francisco declined by $27 \% .^{23}$

Despite these changes to opioid prescribing for pain management in recent years, pain treatment satisfaction, especially among those with CNCP and substance use in safety-net settings, is poorly understood. PCPs and policy makers are compelled to balance patient safety in limiting excessive opioid prescriptions and patient-centered outcomes like satisfaction when developing and implementing interventions in marginalized populations. To better characterize factors associated with pain treatment satisfaction, we conducted an exploratory cross-sectional analysis to characterize pain treatment satisfaction and related factors in a cohort of patients with a history of illicit substance use on long-term opioids for CNCP. Understanding these factors can help PCPs and policy makers tailor pain management strategies and create patient-centered approaches to care.

\section{Methods}

\section{Study Design and Population}

We conducted an exploratory cross-sectional analysis using baseline data from the Cohort study of Opioids, Pain, and Safety IN an era of changinG policy (COPING), a longitudinal cohort study measuring changes in functional status, pain levels, and substance use during a period of changes in opioid prescribing guidelines. From March 2017 to March 2019, we enrolled 300 English-speaking adults receiving primary care through the SFHN who had CNCP, had been on chronic opioid therapy (defined as being prescribed >20 morphine milligram equivalents $[\mathrm{MME}]$ of opioid therapy daily for at least 3 of the preceding 12 months $^{24}$ ), and had prior or active substance use (ie, use of nonmedical opioids, cocaine, or methamphetamine). The SFHN includes 12 adult, urban primary care clinics: 3 clinics are housed within a university-affiliated public hospital, and 9 are community-based clinics serving populations with varying demographics across San Francisco. We oversampled patients with HIV by ensuring we recruited at least 100 patients with HIV, as people living with HIV face unique pain conditions and high rates of substance use. ${ }^{25}$ Each clinic has a registry of patients on opioid therapy for CNCP, which we reviewed to identify patients who had received opioid therapy for at least 3 of the past 12 months and to determine their HIV status. We then contacted primary care providers for permission to call potentially eligible patients to perform a prescreen interview, where we asked about lifetime or active illicit substance use. We invited eligible patients for an enrollment visit.

In the parent COPING study, participants completed a baseline and up to four subsequent, annual visits. Each annual visit included a computerassisted personal interview (CAPI), clinical examination testing, and urine toxicology testing. We also queried participants' electronic medical records 
for information on medical conditions and prescriptions. The present study includes only data from the baseline study visit and chart review. Participants provided written informed consent for study participation. This study was approved by the University of California, San Francisco Institutional Review Board (\#15-18274).

\section{Self-Reported Measures}

Self-reported measures were collected by CAPI, where study staff read questions to the participant and recorded responses using a computer. CAPI interview measures included demographics; mental health comorbidities; characteristics of pain; pharmacologic and nonpharmacologic pain treatments received; past-year use of alcohol, tobacco, and other substances; and lifetime history of substance use treatment and overdose. We asked about cannabis use as a pain treatment rather than assessing as a recreational substance, as the majority of individuals with CNCP use cannabis to treat pain rather than for recreational purposes. $^{26}$

We asked individuals for their gender and sex assigned at birth. Participants could select "Female," "Male," "Transgender," "Other," or "Decline to answer" for gender, and "Female," "Male," "Intersex," or "Decline to answer" for sex assigned at birth. Using the 2-step approach, ${ }^{27}$ we categorized gender as cisgender male (assigned male at birth, male gender), cisgender female (assigned female at birth, female gender), or gender minority person (if gender response was transgender, another gender, or if gender response was female with assigned male at birth or gender response was male with assigned female at birth). We combined race/ethnicity into categories of non-Hispanic White, non-Hispanic Black, Hispanic/Latinx, or other race/ethnicity, and we collapsed education and income assessments due to small counts in some categories.

Participants screened positive for post-traumatic stress disorder (PTSD) if they answered "yes" to $\geq 3$ Primary Care PTSD Screen questions. ${ }^{28}$ Participants screened positive for depression if scores were $\geq 10$ on the Patient Health Questionnaire-8 depression scale (PHQ-8). ${ }^{29}$ The PHQ-8 has identical cut points to the 9-item version, the PHQ-9, ${ }^{30}$ and is preferentially used over the PHQ-9 in clinical research studies. ${ }^{30}$ We screened for psychological distress using the Brief Symptom Inventory (BSI18). ${ }^{31-33}$ BSI scores $\geq 63$ for either the global or 2 or more of the BSI subscales were considered clinically significant for distress. ${ }^{31-33}$ The BSI is validated only for cisgender populations, which did not describe all of our study population.

We asked about chronic pain severity and catastrophizing using (1) average pain in the past 3 months (both scored from 0 [no pain] to 10 [worst possible pain]) and (2) the Pain Catastrophizing Scale (PCS). ${ }^{34}$ PCS is a validated assessment for pain catastrophizing, a cognitive-affective response to anticipated or realized pain associated with worse pain outcomes; we considered a score of $\geq 30$ clinically significant. ${ }^{35,36} \mathrm{We}$ assessed for lifetime and past-year pain treatments, including pharmacologic (opioid and nonopioid medications) and nonpharmacologic (eg, local injections, chiropractic care, physical/occupational therapy, individual or group behavioral counseling, acupuncture, and massage therapy) treatments.

After assessing past-year pain treatments, we asked participants, "Think about all the treatment you have received for pain in the past year. How satisfied are you with the treatment that you received?" Participants responded on a 5-point Likert scale, and we grouped responses into low ("Not at all satisfied" or "Slightly satisfied"), moderate ("Moderately satisfied"), and high satisfaction ("Quite a bit satisfied" or "Extremely satisfied") based on the distribution of the sample. The measure was adopted from several longitudinal studies measuring opioid use and misuse among urban, safety-net primary care patients with $\mathrm{CNCP}$ and a history of substance use. ${ }^{16,37,38}$

\section{Clinical Examination Measures}

We used a cold pressor test (CPT) to measure participants' cold pain threshold and tolerance (in seconds). Cold pain threshold was defined as the amount of time until a participant reported pain after submerging a hand in a $2.0^{\circ} \mathrm{C}$ water bath, and cold pain tolerance was the total time before the participant felt the need to withdraw the hand. ${ }^{39,40}$ We screened participants for neuropathic pain using a Douleur Neuropathique 4 (DN4) score of $\geq 4$. The DN4 combined CAPI items with examination findings of numbness, allodynia, or hypoesthesia. ${ }^{3}$ We used an adapted mini-physical performance test (mPPT) to assess for physical function, ${ }^{41}$ a validated 4-item test evaluating ability to perform four physical tasks (ie, chair rise, picking an item up from the floor, 50-foot walk test, and standing balance). 
Due to participants consistently being unable to pick up a penny, study staff adapted the measure to picking up a marker to increase the variation in functional status scores. Participants completed the mPPT twice during the baseline visit, and we interpreted the test as positive for functional impairment if the highest score was $\leq 11^{41}$

\section{Medical Chart Review}

We reviewed medical charts to collect data on the presence of clinical comorbidities (ie, HIV and hepatitis $\mathrm{C}[\mathrm{HCV}])$. We collected data on $\mathrm{HCV}$ given frequent comorbidity with substance use and possible relationships to chronic pain conditions. We also collected data on any prescribed medications for CNCP, including opioids. ${ }^{42} \mathrm{We}$ did not include data on opioids (eg, buprenorphine or methadone) prescribed solely for opioid use disorder treatment as noted in the chart. We calculated prescribed MME at enrollment and categorized MME to align with CDC guidelines. ${ }^{43,44} \mathrm{We}$ defined opioid discontinuations (stopped for at least 3 months without reinitiation), decreases ( $\geq 30 \%$ reduction in MME without discontinuation), and increases ( $\geq 30 \%$ increase in MME) in the past year. ${ }^{45} \mathrm{We}$ recorded exposure to opioid stewardship measures, including controlled substance agreements, urine drug screen (UDS) completed in past year, and receipt of naloxone prescription. Within SFHN, it is advised that all participants receiving opioid therapy for any indication be prescribed naloxone.

\section{Urine Drug Screen}

Participants completed a UDS immunoassay at baseline; indeterminate results were rare and interpreted as negative (see Appendix 1 for list of detectable substances). Among participants with UDS positive for opioids, we further categorized polysubstance use with opioids (excluding cannabis) on UDS as opioids only (including methadone or buprenorphine), opioids with stimulants only (ie, cocaine or amphetamines), and opioids combined with other substances, which were grouped together due to small sample sizes across response categories. We examined whether UDS was consistent with being prescribed detectable opioid therapy, defined as being prescribed $\geq 20 \mathrm{MME}$ of opioid therapy based on chart review (excluding fentanyl therapy, as fentanyl was not detectable on the UDS used). Participants who did not complete UDS were excluded from UDS analyses.

\section{Statistical Analysis}

We used descriptive statistics to summarize baseline demographics; comorbidities; alcohol, tobacco, and other substance use history; UDS; and pain severity, characteristics, and management. We compared characteristics across pain treatment satisfaction levels. We used ANOVA testing for normally distributed continuous variables and the KruskalWallis test for skewed variables. For categorical variables, we used Pearson's chi-square test, and Fischer's exact test for expected cell counts less than five.

We assessed for factors associated with pain treatment satisfaction using a multivariable ordinal regression model including a priori defined independent variables (age and gender based on the 2step gender assessment) and predictors from bivariate analyses with a $P$ value of $<0.05$ (see Appendix 2 for details on data analysis). We did not include being prescribed hydromorphone or buprenorphine in the multivariable model due to small sample sizes. All analyses were done in Stata Version 16.0 (Stata Corp, College Station, TX).

\section{Results}

\section{Participant Characteristics}

We enrolled 300 individuals with a mean age of 57.5 (SD \pm 8.1$)$ years. The majority were cisgender men $(60 \%)$, and nearly half were non-Hispanic Black (44\%). Most (77\%) had experienced homelessness at some point, $35 \%$ had HIV due to oversampling, and $50 \%$ had current or prior $\mathrm{HCV}$ infection. Most (78\%) reported past-year substance use, most commonly tobacco (56\%), alcohol (51\%), cocaine $(24 \%)$, methamphetamine $(22 \%)$, and heroin (17\%). About a fifth (19\%) reported a history of prior opioid overdose (Table 1).

\section{Pain and Pain Treatment Characteristics}

The median score for "average pain in the past 3 months" was 7 (IQR 6 to 9). Most participants (83\%) were being prescribed opioid therapy. A fifth of participants had a $\geq 30 \%$ opioid dose reduction in the past year (18\%), and $14 \%$ had a past-year discontinuation. The majority (73\%) reported being prescribed at least one nonopioid medication for CNCP, most commonly gabapentinoids (35\%), acetaminophen or nonsteroidal anti-inflammatory drugs (NSAIDs) (31\%), and/or cannabis (31\%) (Table 2). Out of 299 participants who responded 
Table 1. Demographic and Substance Use Characteristics of Safety-Net Patients on or Recently on Long-Term Opioid Therapy for Chronic Noncancer Pain, by Level of Satisfaction with Pain Treatment*

\begin{tabular}{|c|c|c|c|c|c|}
\hline $\begin{array}{l}\text { Characteristic, Median (IQR) } \\
\text { or N }(\%)\end{array}$ & $\begin{array}{l}\text { All Participants } \\
\quad(\mathrm{n}=300)\end{array}$ & $\begin{array}{l}\text { Low Satisfaction } \\
\qquad(\mathrm{n}=116)\end{array}$ & $\begin{array}{l}\text { Moderate Satisfaction } \\
\quad(\mathrm{n}=85)\end{array}$ & $\begin{array}{l}\text { High Satisfaction } \\
\qquad(\mathrm{n}=98)\end{array}$ & $P$ Value \\
\hline Age & $57.5( \pm 8.1)$ & $56.9(9.1)$ & $57.6(7.9)$ & $58.1(7.2)$ & 0.57 \\
\hline \multicolumn{6}{|l|}{ Gender } \\
\hline Cisgender female & $101(34 \%)$ & $52(51 \%)$ & $25(25 \%)$ & $24(24 \%)$ & \multirow[t]{3}{*}{0.002} \\
\hline Cisgender male & $182(60 \%)$ & $63(35 \%)$ & $54(30 \%)$ & $64(35 \%)$ & \\
\hline Gender minority person & $17(6 \%)$ & $1(6 \%)$ & $6(35 \%)$ & $10(59 \%)$ & \\
\hline \multicolumn{6}{|l|}{ Race/ethnicity $^{\dagger}$} \\
\hline Non-Hispanic White & $95(32 \%)$ & $34(36 \%)$ & $28(30 \%)$ & $32(34 \%)$ & \multirow[t]{4}{*}{0.26} \\
\hline Non-Hispanic Black & $131(44 \%)$ & $55(42.0 \%)$ & $36(28 \%)$ & $40(31 \%)$ & \\
\hline Hispanic/Latinx & $33(11 \%)$ & $17(52 \%)$ & $5(15 \%)$ & $11(33 \%)$ & \\
\hline Mixed or other & $40(13 \%)$ & $10(24 \%)$ & $15(38 \%)$ & $15(37 \%)$ & \\
\hline \multicolumn{6}{|l|}{ Education } \\
\hline Some high school or less & $74(25 \%)$ & $27(37 \%)$ & $21(29 \%)$ & $25(34 \%)$ & \multirow[t]{3}{*}{0.95} \\
\hline GED/some college & $169(56 \%)$ & $66(39 \%)$ & $50(30 \%)$ & $53(31 \%)$ & \\
\hline $\begin{array}{l}\text { Vocational training/college or } \\
\text { higher }\end{array}$ & $57(19 \%)$ & $23(40 \%)$ & $14(25 \%)$ & $20(35 \%)$ & \\
\hline \multicolumn{6}{|l|}{ Income $^{\dagger}$} \\
\hline$<\$ 9999$ & $56(19 \%)$ & $22(39 \%)$ & $18(32 \%)$ & $16(29 \%)$ & \multirow[t]{3}{*}{0.93} \\
\hline$\$ 10,000-\$ 19,999$ & $193(65 \%)$ & $75(39 \%)$ & $52(27 \%)$ & $66(34 \%)$ & \\
\hline$>\$ 20,000$ & $49(16 \%)$ & $18(38 \%)$ & $14(29 \%)$ & $16(33 \%)$ & \\
\hline \multicolumn{6}{|l|}{ Comorbidities } \\
\hline Ever homeless & $230(77 \%)$ & $93(41 \%)$ & $69(30 \%)$ & $67(29 \%)$ & 0.06 \\
\hline HIV positive & $105(35 \%)$ & $32(31 \%)$ & $30(29 \%)$ & $43(41 \%)$ & 0.05 \\
\hline History of hepatitis $\mathrm{C}$ infection & $151(50 \%)$ & $60(40 \%)$ & $46(31 \%)$ & $44(29 \%)$ & 0.42 \\
\hline $\begin{array}{l}\text { Brief Symptom Inventory (BSI) } \\
\quad \text { score } \geq 63^{\ddagger}\end{array}$ & $83(28 \%)$ & $36(43 \%)$ & $29(35 \%)$ & $18(22 \%)$ & 0.006 \\
\hline $\begin{array}{l}\text { Patient Health Questionnaire-8 } \\
\text { (PHQ-8) Depression Scale } \geq 10\end{array}$ & $83(28 \%)$ & $38(46 \%)$ & $28(34 \%)$ & $17(21 \%)$ & 0.02 \\
\hline $\begin{array}{l}\text { Post-traumatic stress disorder } \\
\quad \text { screen } \geq 3\end{array}$ & $99(33 \%)$ & $45(46 \%)$ & $31(32 \%)$ & $22(23 \%)$ & 0.03 \\
\hline $\begin{array}{l}\text { Mini-physical performance test } \\
\quad(\mathrm{mPPT}) \leq 11^{\S}\end{array}$ & $145(52 \%)$ & $60(41 \%)$ & $41(28 \%)$ & $44(30 \%)$ & 0.72 \\
\hline \multicolumn{6}{|l|}{$\begin{array}{l}\text { Self-reported substance use in past } \\
\text { year } \|\end{array}$} \\
\hline No drugs, alcohol, or tobacco & $66(22 \%)$ & $21(32 \%)$ & $16(24 \%)$ & $29(44 \%)$ & 0.09 \\
\hline Alcohol & $154(51 \%)$ & $61(40 \%)$ & $48(31 \%)$ & $44(29 \%)$ & 0.27 \\
\hline Tobacco & $169(56 \%)$ & $74(44 \%)$ & $50(30 \%)$ & $44(26 \%)$ & 0.02 \\
\hline Any illicit substances & $121(40 \%)$ & $52(43 \%)$ & $34(28 \%)$ & $35(29 \%)$ & 0.40 \\
\hline - Heroin & $51(17 \%)$ & $25(49 \%)$ & $12(24 \%)$ & $14(28 \%)$ & 0.26 \\
\hline - Methamphetamine or speed & $66(22 \%)$ & $28(42 \%)$ & $21(32 \%)$ & $17(26 \%)$ & 0.39 \\
\hline - Cocaine or crack cocaine & $72(24 \%)$ & $34(47 \%)$ & $21(29 \%)$ & $17(24 \%)$ & 0.12 \\
\hline - Other ${ }^{\pi}$ & $18(6 \%)$ & $7(39 \%)$ & $5(28 \%)$ & $6(33 \%)$ & 0.99 \\
\hline History of substance use treatment & $203(68 \%)$ & $79(39 \%)$ & $58(29 \%)$ & $65(32 \%)$ & 0.95 \\
\hline History of prior overdose & $56(19 \%)$ & $27(48 \%)$ & $13(23 \%)$ & $16(29 \%)$ & 0.27 \\
\hline
\end{tabular}

IQR, interquartile range.

${ }^{*}$ One participant did not respond to the pain treatment satisfaction question.

${ }^{\dagger}$ One participant declined to state their race, and two participants declined to state their income.

${ }^{\ddagger}$ BSI scores only interpretable for 282 cisgender participants and considered positive if either global score or two subscale scores were $\geq 63$.

${ }_{\mathrm{mPPT}}$ scores were conducted in 277 participants at baseline. We used a cut-off score of 11 or lower as evidence of functional impairment.

"Measure does not include cannabis use.

"Other substances including inhalants or hallucinogens. 
to the question about pain treatment satisfaction, $39 \%$ reported low, 28\% moderate, and 33\% high satisfaction.

\section{Urine Drug Screen Results}

Most participants $(92 \%, \mathrm{n}=276)$ completed a UDS; $65 \%$ were positive for opioids. Forty-seven participants $(17 \%)$ were prescribed opioid therapy that should have been detectable on UDS and screened opioid negative; 17 of whom were prescribed 90 MME or higher (Table 3).

\section{Unadjusted Analyses of Factors Associated with Pain Treatment Satisfaction}

In unadjusted analyses, factors with a higher proportion of low pain treatment satisfaction levels included cisgender female gender, tobacco use, past-year opioid discontinuation, not taking any medications for pain, and screening positive for depression, PTSD, and psychological distress on the BSI (Table 1). Participant factors with higher levels of treatment satisfaction were living with HIV (Table 1) and using cannabis for pain (Table 2 ). UDS being positive for cannabis was not associated with satisfaction, and only $60 \%(\mathrm{n}=64)$ of these individuals were being prescribed cannabis for pain. Buprenorphine detected on UDS and selfreport of hydromorphone therapy did differ significantly though sample sizes were small. Satisfaction levels did not differ significantly by primary care clinic (data not shown).

\section{Multivariable Analyses of Factors Associated with Pain Treatment Satisfaction}

In multivariable analysis, living with HIV (adjusted odds ratio [AOR] 1.6, 95\%, CI, 1.0-2.7) and using prescribed cannabis for pain (AOR 1.7, 95\% CI, 1.0-2.7) were associated with higher satisfaction. Screening positive for PTSD (AOR 0.6, 95\% CI, $0.3-0.9)$, higher average pain in the past 3 months (AOR 0.9, 95\% CI, 0.8-1.0), tobacco use (AOR 0.6, 95\% CI, 0.4-0.9), and past-year opioid discontinuation (AOR 0.4, 95\% CI, 0.2-0.9) were associated with lower satisfaction (Table 4).

\section{Discussion}

Among patients with CNCP on recent opioid therapy and a history of illicit substance use recruited from an urban, safety-net health system, more than one third were not at all or only slightly satisfied with their pain treatment, and opioid discontinuations were associated with lower pain treatment satisfaction. Due to our cross-sectional design, it is unknown whether opioid discontinuations led to dissatisfaction, or if treatment dissatisfaction led to discontinuation. However, this finding aligns with growing concerns with consequences of discontinuing opioid therapy in patients with CNCP, especially in those with substance use. ${ }^{46,47}$ Despite a lack of evidence supporting long-term opioid efficacy in CNCP treatment and some evidence suggesting improved pain scores with opioid tapering, ${ }^{48,49}$ recent studies have cited potential harms of opioid discontinuations, including increased suicidal ideation from uncontrolled pain, return or initiation of illicit drug use leading to increased overdose risk and death, and increased emergency department visits and hospitalizations from adverse health events. $^{50-56}$

While we do not know whether participants consented to opioid discontinuations, mounting pressure from regulators to curb opioid prescriptions suggests many were likely provider-directed. ${ }^{18,21,24,45}$ Even as discontinuations likely had justifications in prioritizing safety, our finding highlights the need for providers and policy makers to further examine the potential negative impacts of opioid prescribing policy changes. Using thoughtful, patient-centered strategies during opioid discontinuations may also help alleviate some of these negative impacts, such as avoiding rapid or sudden discontinuations, engaging patients as much as possible in decision making, ensuring adequate access to comprehensive, multimodal treatment for both pain and cooccurring substance use disorders, and providing care with multidisciplinary teams to broadly address patient concerns. ${ }^{6,57}$ Only about a third of patients at most were taking gabapentinoids or other neuropathic medications, which were increasingly recommended at the time of this study, and further research is needed to explore if increasing use of these medications contributes to treatment satisfaction. ${ }^{58}$

PTSD and tobacco use were associated with lower treatment satisfaction. PTSD and related mental health diagnoses are challenging to treat in primary care and are associated with increased risk of opioid use disorder and overdose. ${ }^{1-34,59,60}$ Using trauma-informed approaches in CNCP treatment may help address quality of care and satisfaction. Including mental health professionals in care as 
Table 2. Characteristics of Pain Treatment for Safety-Net Patients on or Recently on Long-Term Opioid Therapy for Chronic Noncancer Pain, by Level of Satisfaction with Pain Treatment*

\begin{tabular}{|c|c|c|c|c|c|}
\hline $\begin{array}{l}\text { Characteristic, Median (IQR) or } \\
\mathrm{n}(\%)\end{array}$ & $\begin{array}{l}\text { All Participants } \\
\quad(\mathrm{n}=300)\end{array}$ & $\begin{array}{l}\text { Low Satisfaction } \\
\qquad(\mathrm{n}=116)\end{array}$ & $\begin{array}{l}\text { Moderate Satisfaction } \\
\qquad(\mathrm{n}=85)\end{array}$ & $\begin{array}{l}\text { High Satisfaction } \\
\qquad(\mathrm{n}=98)\end{array}$ & $P$ Value \\
\hline \multicolumn{6}{|l|}{ Pain characteristics } \\
\hline $\begin{array}{l}\text { Pain on average in the past } \\
3 \text { months }\end{array}$ & $7(6$ to 9$)$ & $8(7$ to 9$)$ & $7(6$ to 8$)$ & $7(6$ to 9$)$ & 0.015 \\
\hline $\begin{array}{l}\text { Pain Catastrophizing } \\
\text { Scale } \geq 30\end{array}$ & $96(32 \%)$ & $45(47 \%)$ & $27(28 \%)$ & $23(24 \%)$ & 0.06 \\
\hline Cold pressor threshold score & $7.5(5.1$ to 11.5$)$ & $7.7(5.2$ to 11.6$)$ & $7.6(5.3$ to 11.7$)$ & $7.0(4.3$ to 11.0$)$ & 0.61 \\
\hline Cold pressor tolerance score & $10.9(7.0$ to 17.7$)$ & $11.1(6.9$ to 17.7$)$ & $11.5(7.5$ to 17.7$)$ & 10.5 (6.6 to 18.6$)$ & 0.74 \\
\hline $\begin{array}{l}\text { Douleur Neuropathique } 4 \\
\text { (Neuropathic Pain } \\
\text { Assessment) } \geq 4^{\dagger}\end{array}$ & $161(56 \%)$ & $66(41 \%)$ & $48(30 \%)$ & $46(29 \%)$ & 0.23 \\
\hline $\begin{array}{l}\text { Prescribed opioid MME at } \\
\text { baseline }\end{array}$ & $60(17.5$ to 180$)$ & $43(6$ to 171$)$ & $60(23$ to 150$)$ & $60(20$ to 222$)$ & 0.22 \\
\hline $0 \mathrm{MME}$ & $50(17 \%)$ & $27(54 \%)$ & $11(22 \%)$ & $12(24 \%)$ & 0.25 \\
\hline 1 to $19 \mathrm{MME}$ & $25(8 \%)$ & $8(32 \%)$ & $6(24 \%)$ & $11(44 \%)$ & \\
\hline 20 to $89 \mathrm{MME}$ & $102(34 \%)$ & $38(37 \%)$ & $33(32 \%)$ & $31(31 \%)$ & \\
\hline 90 to $199 \mathrm{MME}$ & $57(19 \%)$ & $19(33 \%)$ & $20(35 \%)$ & $18(32 \%)$ & \\
\hline$\geq 200 \mathrm{MME}$ & $66(22 \%)$ & $24(37 \%)$ & $15(23 \%)$ & $26(40 \%)$ & \\
\hline Max MME in past year & 112.5 (36.0 to 246.3$)$ & 105 (40 to 270$)$ & $90(36$ to 216$)$ & $120(30$ to 270$)$ & 0.77 \\
\hline $\begin{array}{l}\text { MME decreased }>30 \% \text { and } \\
\text { not stopped in past year }\end{array}$ & $53(18 \%)$ & $24(45 \%)$ & $11(21 \%)$ & $18(34 \%)$ & 0.36 \\
\hline $\begin{array}{l}\text { MME increased }>30 \% \text { in } \\
\text { past year }\end{array}$ & $42(14 \%)$ & $13(31 \%)$ & $14(33 \%)$ & $15(36 \%)$ & 0.52 \\
\hline $\begin{array}{l}\text { Opioid(s) discontinued in past } \\
\text { year }\end{array}$ & $43(14 \%)$ & $26(61 \%)$ & $9(21 \%)$ & $8(19 \%)$ & 0.006 \\
\hline \multicolumn{6}{|l|}{$\begin{array}{l}\text { Self-reported prescribed } \\
\text { opioids }\end{array}$} \\
\hline Oxycodone, hydrocodone & $167(56 \%)$ & $60(36 \%)$ & $46(28 \%)$ & $60(36 \%)$ & 0.36 \\
\hline Hydromorphone & $6(2 \%)$ & $1(16 \%)$ & $0(0 \%)$ & $5(83 \%)$ & 0.03 \\
\hline Morphine & $81(27 \%)$ & $25(31 \%)$ & $28(35 \%)$ & $27(34 \%)$ & 0.19 \\
\hline Methadone & $54(18 \%)$ & $26(48 \%)$ & $16(30 \%)$ & $12(22 \%)$ & 0.15 \\
\hline Fentanyl & $12(4 \%)$ & $3(25 \%)$ & $5(42 \%)$ & $4(33 \%)$ & 0.53 \\
\hline Codeine & $21(7 \%)$ & $6(29 \%)$ & $7(33 \%)$ & $8(38 \%)$ & 0.61 \\
\hline Other opioid & $6(2 \%)$ & $1(17 \%)$ & $4(68 \%)$ & $1(17 \%)$ & 0.11 \\
\hline No opioids & $38(13 \%)$ & $22(58 \%)$ & $6(16 \%)$ & $10(26 \%)$ & 0.03 \\
\hline \multicolumn{6}{|l|}{$\begin{array}{l}\text { Self-reported nonopioid } \\
\text { medications }\end{array}$} \\
\hline $\begin{array}{l}\text { Acetaminophen or } \\
\text { nonsteroidal anti- } \\
\text { inflammatory drugs } \\
\text { (NSAIDs) }\end{array}$ & $94(31 \%)$ & $36(39 \%)$ & $29(31 \%)$ & $28(30 \%)$ & 0.72 \\
\hline $\begin{array}{l}\text { Gabapentinoids (gabapentin, } \\
\text { pregabalin) }\end{array}$ & $105(35 \%)$ & $38(37 \%)$ & $36(35 \%)$ & $30(29 \%)$ & 0.21 \\
\hline Cannabis (prescribed) & $93(31 \%)$ & $25(27 \%)$ & $30(33 \%)$ & $37(40 \%)$ & 0.02 \\
\hline Muscle relaxants & $43(14 \%)$ & $10(24 \%)$ & $17(41 \%)$ & $15(36 \%)$ & 0.07 \\
\hline $\begin{array}{l}\text { Other neuropathic } \\
\text { medications }\end{array}$ & $29(10 \%)$ & $10(36 \%)$ & $7(25 \%)$ & $11(39 \%)$ & 0.74 \\
\hline $\begin{array}{l}\text { Topical medications } \\
\text { (lidocaine, capsaicin) }\end{array}$ & $28(9 \%)$ & $14(50 \%)$ & $4(14 \%)$ & $10(36 \%)$ & 0.20 \\
\hline $\begin{array}{l}\text { Other medications or do not } \\
\text { remember }\end{array}$ & $12(4 \%)$ & $4(33 \%)$ & $4(33 \%)$ & $4(33 \%)$ & 0.93 \\
\hline No nonopioid medications & $80(27 \%)$ & $36(45 \%)$ & $17(21 \%)$ & $27(34 \%)$ & 0.21 \\
\hline
\end{tabular}


Table 2. Continued

\begin{tabular}{|c|c|c|c|c|c|}
\hline $\begin{array}{l}\text { Characteristic, Median (IQR) or } \\
\mathrm{n}(\%)\end{array}$ & $\begin{array}{l}\text { All Participants } \\
\qquad(\mathrm{n}=300)\end{array}$ & $\begin{array}{l}\text { Low Satisfaction } \\
\qquad(\mathrm{n}=116)\end{array}$ & $\begin{array}{l}\text { Moderate Satisfaction } \\
\qquad(\mathrm{n}=85)\end{array}$ & $\begin{array}{l}\text { High Satisfaction } \\
\qquad(\mathrm{n}=98)\end{array}$ & $P$ Value \\
\hline \multicolumn{6}{|l|}{ Nonmedication treatments } \\
\hline No medications & $10(3 \%)$ & $8(80 \%)$ & $1(10 \%)$ & $1(10 \%)$ & 0.04 \\
\hline Local injections & $55(18 \%)$ & $26(47 \%)$ & $15(27 \%)$ & $14(26 \%)$ & 0.30 \\
\hline Chiropractic care & $24(8 \%)$ & $12(52 \%)$ & $4(17 \%)$ & $7(30 \%)$ & 0.34 \\
\hline $\begin{array}{l}\text { Physical or occupational } \\
\text { therapy }\end{array}$ & $86(29 \%)$ & $35(41 \%)$ & $29(34 \%)$ & $22(26 \%)$ & 0.20 \\
\hline Acupuncture & $49(16 \%)$ & $17(35 \%)$ & $12(25 \%)$ & $19(40 \%)$ & 0.55 \\
\hline Massage therapy & $59(20 \%)$ & $20(35 \%)$ & $15(26 \%)$ & $23(40 \%)$ & 0.46 \\
\hline $\begin{array}{l}\text { Group or individual } \\
\text { behavioral counseling }\end{array}$ & $53(18 \%)$ & $20(38 \%)$ & $18(34 \%)$ & $15(28 \%)$ & 0.58 \\
\hline \multicolumn{6}{|l|}{$\begin{array}{l}\text { Opioid stewardship } \\
\text { interventions }\end{array}$} \\
\hline $\begin{array}{l}\text { Pain agreement documented } \\
\text { in chart }\end{array}$ & $208(69 \%)$ & $80(39 \%)$ & $62(30 \%)$ & $66(32 \%)$ & 0.70 \\
\hline $\begin{array}{l}\text { Naloxone prescribed in } \\
\text { chart }\end{array}$ & $182(61 \%)$ & $75(41 \%)$ & $52(29 \%)$ & $55(30 \%)$ & 0.44 \\
\hline $\begin{array}{l}\text { Urine drug screen done in } \\
\text { the past year }\end{array}$ & $247(82 \%)$ & $100(40 \%)$ & $68(28 \%)$ & $79(32 \%)$ & 0.42 \\
\hline
\end{tabular}

IQR, interquartile range; MME, Morphine milligram equivalents.

*One participant did not respond to the pain treatment satisfaction question.

${ }^{\dagger}$ There were 14 participants who did not complete neuropathic pain assessment.

${ }^{\ddagger}$ Other neuropathic medications including tricyclic antidepressants (amitriptyline, desipramine), serotonin-norepinephrine reuptake inhibitors (duloxetine, venlafaxine), migraine medications (sumatriptan, fioricet, midrin, ergotamine, topiramate), and antiseizure medications (valproic acid).

appropriate could be one way of incorporating trauma-informed approaches and increasing multidisciplinary collaboration. Our results for tobacco could be due to the pharmacologic effect of chronic tobacco use, leading to vascular disorders or other conditions that worsen chronic pain and thus treatment satisfaction. ${ }^{61-63}$ Alternatively, tobacco use could represent a marker for structural factors associated with worse pain outcomes, such as socioeconomic instability and higher rates of mental and physical health comorbidities; tobacco use also could be a coping mechanism in the face of all of these factors. ${ }^{63,64}$ Both PTSD and tobacco use may indicate a need for more targeted efforts to improve pain treatment and other quality-of-care markers.

Higher pain treatment satisfaction among participants living with HIV was surprising, given higher rates of comorbid mental health and substance use disorders in those living with HIV and difficult-totreat pain syndromes such as HIV-related neuropathy. ${ }^{13,25,65,66}$ Reasons are likely multifactorial: HIV care is more often provided in multidisciplinary teams with access to pharmacists, nurses, mental health providers, in addition to PCPs providing HIV care. ${ }^{67}$ Care from multidisciplinary teams may lead to higher use of multimodal pain treatments that improve pain treatment satisfaction. ${ }^{67,68}$ Although most multimodal pain treatments offered (eg, acupuncture, behavioral therapy) in our study were not significantly associated with satisfaction, the proportions of participants who accessed these therapies in this study were small. Prior studies exploring pain management in this population have, in fact, reported effectiveness of multimodal pain treatments in managing pain, suggesting these services may offer benefit if made more available to patients. $6,7,56$

Studies show HIV providers operate within the "HIV paradigm," prioritizing patient engagement, care retention, adherence to therapy, and viral suppression. ${ }^{69,70}$ This paradigm may conflict with conservative opioid prescribing guidelines, and HIV providers may be more likely to continue opioid prescriptions or tolerate problematic substance use out of concern that opioid discontinuation may reduce care retention and worsen HIV outcomes. ${ }^{69}$ HIV providers may also feel more comfortable treating substance use disorders, have longer-term relationships with patients, and be more likely to identify as patient allies or practice trauma- 
Table 3. Results of Urine Drug Screen (UDS) at Study Enrollment for Safety-Net Patients on or Recently on LongTerm Opioid Therapy for Chronic Noncancer Pain $(\mathrm{n}=\mathbf{2 7 6})^{*}$

\begin{tabular}{|c|c|c|c|c|c|}
\hline $\begin{array}{l}\text { Characteristic, Median (IQR) or } \\
\mathrm{n}(\%)\end{array}$ & $\begin{array}{l}\text { All Participants } \\
\quad(\mathrm{n}=300)\end{array}$ & $\begin{array}{l}\text { Low Satisfaction } \\
\quad(\mathrm{n}=116)\end{array}$ & $\begin{array}{l}\text { Moderate Satisfaction } \\
\quad(\mathrm{n}=85)\end{array}$ & $\begin{array}{l}\text { High Satisfaction } \\
\qquad(\mathrm{n}=98)\end{array}$ & $P$ Value \\
\hline \multicolumn{6}{|l|}{ Drugs detected on baseline UDS } \\
\hline No drugs detected & $41(15 \%)$ & $15(37 \%)$ & $12(29 \%)$ & $14(34 \%)$ & 0.93 \\
\hline $\begin{array}{l}\text { Opioids (including methadone } \\
\text { and buprenorphine) }\end{array}$ & $178(65 \%)$ & $71(40 \%)$ & $50(28 \%)$ & $56(32 \%)$ & 0.79 \\
\hline Methadone & $72(26 \%)$ & $33(46 \%)$ & $19(26 \%)$ & $20(28 \%)$ & 0.38 \\
\hline Buprenorphine & $7(3 \%)$ & $0(0 \%)$ & $5(71 \%)$ & $2(29 \%)$ & 0.01 \\
\hline Benzodiazepines & $17(6 \%)$ & $6(35 \%)$ & $4(24 \%)$ & $7(41 \%)$ & 0.76 \\
\hline Cocaine & $58(21 \%)$ & $28(48 \%)$ & $18(31 \%)$ & $12(21 \%)$ & 0.07 \\
\hline $\begin{array}{l}\text { Amphetamines/ } \\
\text { methamphetamine }\end{array}$ & $37(13 \%)$ & $37(35 \%)$ & $30(28 \%)$ & $40(37 \%)$ & 0.37 \\
\hline Tetrahydrocannabinol (THC) & $107(39 \%)$ & $18(49 \%)$ & $12(32 \%)$ & $7(19 \%)$ & 0.14 \\
\hline Other drugs ${ }^{\dagger}$ & $3(1 \%)$ & $0(0 \%)$ & $2(67 \%)$ & $1(3 \%)$ & 0.19 \\
\hline \multicolumn{6}{|l|}{$\begin{array}{l}\text { Opioids in combination with } \\
\text { other drugs }\end{array}$} \\
\hline No opioids & $98(36 \%)$ & $37(38 \%)$ & $26(27 \%)$ & $35(36 \%)$ & 0.13 \\
\hline $\begin{array}{l}\text { Opioids only (including } \\
\text { methadone and } \\
\text { buprenorphine) }\end{array}$ & $117(42 \%)$ & $42(36 \%)$ & $32(28 \%)$ & $42(36 \%)$ & \\
\hline $\begin{array}{l}\text { Opioids and stimulants only } \\
\text { (ie, cocaine or amphetamines) }\end{array}$ & $49(18 \%)$ & $26(53 \%)$ & $15(31 \%)$ & $8(16 \%)$ & \\
\hline $\begin{array}{l}\text { Opioids and other combination } \\
\text { of drugs }{ }^{\ddagger}\end{array}$ & $12(4 \%)$ & $3(25 \%)$ & $3(25 \%)$ & $6(50 \%)$ & \\
\hline \multicolumn{6}{|l|}{$\begin{array}{l}\text { If UDS consistent with } \\
\text { prescribing }^{\S}\end{array}$} \\
\hline UDS consistent with prescribing & $201(74 \%)$ & $82(41 \%)$ & $54(27 \%)$ & $65(32 \%)$ & 0.58 \\
\hline $\begin{array}{l}\text { UDS positive for opioids and } \\
\text { not prescribed detectable } \\
\text { opioid therapy }\end{array}$ & $25(9 \%)$ & $12(48 \%)$ & $6(24 \%)$ & $7(28 \%)$ & \\
\hline $\begin{array}{l}\text { UDS negative for opioids and } \\
\text { prescribed detectable opioid } \\
\text { therapy }\end{array}$ & $47(17 \%)$ & $14(30 \%)$ & $14(30 \%)$ & $19(40 \%)$ & \\
\hline 20 to $89 \mathrm{MME}$ at baseline & $30(11)$ & $11(37 \%)$ & $8(27 \%)$ & $11(37 \%)$ & \\
\hline 90 to $199 \mathrm{MME}$ at baseline & $11(4 \%)$ & $1(9 \%)$ & $4(36 \%)$ & $6(55 \%)$ & \\
\hline$\geq 200 \mathrm{MME}$ at baseline & $6(2 \%)$ & $2(33 \%)$ & $2(33 \%)$ & $2(33 \%)$ & \\
\hline
\end{tabular}

IQR, interquartile range.

*Twenty-four participants did not complete a UDS at baseline.

${ }^{\dagger}$ Other drugs detected include phencyclidine (PCP) or barbiturates.

' Including opioids combined with benzodiazepines, PCP, barbiturates, with or without stimulants such as cocaine or methamphetamine.

${ }^{\S}$ Detectable opioid therapy defined as milligram morphine equivalent (MME) of at least 20 and taking a full agonist or partial opioid excluding fentanyl, which would not return positive on UDS. Two participants were prescribed only fentanyl as their opioid therapy so were excluded from this measure.

informed care, potentially leading to higher pain treatment satisfaction. ${ }^{70,71}$ Future studies should also explore what factors and lessons from HIV care can be extended to non-HIV settings to improve pain treatment satisfaction for all patients living with CNCP.

The association of cannabis use for pain treatment with higher satisfaction is intriguing, especially as UDS being positive for cannabis was not significantly associated with treatment satisfaction. Of those who tested positive for cannabis on UDS, only $60 \%$ were being prescribed cannabis for pain, potentially diluting pain treatment satisfaction response for cannabis. Although there is increasing attention toward cannabis as an opioid-sparing alternative in CNCP treatment, data are mixed. Studies have suggested using cannabis for CNCP is associated with reduced opioid use and improved 
Table 4. Multivariable Analysis of Greater Pain Satisfaction among Safety-Net Patients on or Recently on Long-Term Opioid Therapy for Chronic Noncancer Pain $(\mathrm{n}=299)^{*}$

\begin{tabular}{lcc}
\hline Characteristic & $\begin{array}{c}\text { Adjusted Odds Ratio } \\
(95 \% \text { CI })\end{array}$ & $\begin{array}{c}P \\
\text { Value }\end{array}$ \\
\hline Age (per 10 years) & $1.1(0.8-1.5)$ & 0.49 \\
Gender & & \\
$\quad$ Cisgender female & Ref & \\
$\quad$ Cisgender male & $1.5(0.9-2.4)$ & 0.12 \\
$\quad$ Gender minority person & $30.8(1.9$ to 14.8$)$ & 0.20 \\
HIV-positive & $1.6(1.0$ to 2.7$)$ & 0.04 \\
Depression & $0.9(0.5-1.7)$ & 0.87 \\
Brief Symptom Inventory (BSI) & $0.8(0.5-1.5)$ & 0.51 \\
$\quad$ score $\geq 63$ & & \\
Post-traumatic stress disorder & $0.6(0.3-0.9)$ & 0.02 \\
Tobacco use & $0.6(0.4-0.9)$ & 0.02 \\
Average pain in past 3 months & $0.9(0.8-1.0)$ & 0.007 \\
Opioids discontinued & $0.4(0.2-0.9)$ & 0.02 \\
Medical cannabis use & $1.7(1.0$ to 2.7$)$ & 0.03 \\
\hline
\end{tabular}

CI, confidence interval.

*One participant did not respond answer the pain treatment satisfaction question.

pain treatment, while other studies found no improvements. ${ }^{72-77}$ Qualitative research in safetynet settings found patients use cannabis for CNCP when opioid prescriptions are limited and express concerns about cannabis dosing and addictive potential, while providers desire more education on how to advise patients. ${ }^{78}$ Long-term psychoactive effects of cannabis in this population with a high prevalence of mental health conditions are also understudied. Much work is needed to determine the efficacy and safety of medical cannabis use in the treatment of CNCP. ${ }^{79}$

Our study had several limitations. Generalizability of these results to other populations is also unclear, as San Francisco may be unique especially in regard to communities living with HIV, though it is also likely other safety-net populations with high rates of substance use also have higher prevalence of HIV. The parent COPING study is also one of few prospective studies examining the effects of changing opioid policy on safety-net patients, and this work provides an impetus for examinations of the impact of opioid prescribing policies. We also did not measure quality of CNCP care or barriers to care access. However, because all participants were SFHN patients who are publicly insured or covered by the local safety-net health care program, barriers to accessing care in this population were likely similar. Our study did not measure patient perceptions of specific pain treatment or the quality of interpersonal interactions with providers during treatment, and future qualitative studies could help explore these domains of pain treatment.

\section{Conclusions}

We found recent opioid discontinuations were associated with less pain treatment satisfaction, highlighting the need for patient-centered approaches balancing patient safety and treatment satisfaction. We identified factors associated with worse (eg, PTSD, tobacco use) and superior (eg, HIV, cannabis use) pain treatment satisfaction, representing potential targets for research and practice improvement as we attempt to safely reduce long-term opioid reliance for CNCP.

We would like to acknowledge the contributions of the research team at the Center on Substance Use and Health and the participants who provided their time and life experience for this study.

To see this article online, please go to: http://jabfm.org/content/ 34/6/1082.full.

\section{References}

1. Institute of Medicine (US) Committee on Advancing Pain Research, Care, and Education. Relieving pain in America: a blueprint for transforming prevention, care, education, and research. Washington: National Academies Press; 2011. Available from: http://www.ncbi.nlm.nih.gov/books/NBK91497/.

2. Dahlhamer J, Lucas J, Zelaya C, et al. Prevalence of chronic pain and high-impact chronic pain among adults-United States, 2016. MMWR Morb Mortal Wkly Rep 2018;67:1001-6.

3. Bouhassira D, Lantéri-Minet M, Attal N, Laurent B, Touboul C. Prevalence of chronic pain with neuropathic characteristics in the general population. Pain 2008;136:380-7.

4. Liebschutz JM, Saitz R, Weiss RD, et al. Clinical factors associated with prescription drug use disorder in urban primary care patients with chronic pain. J Pain 2010;11:1047-55.

5. Hurstak EE, Kushel M, Chang J, et al. The risks of opioid treatment: perspectives of primary care practitioners and patients from safety-net clinics. Subst Abuse 2017;38:213-21.

6. Chao MT, Hurstak E, Leonoudakis-Watts K, et al. Patient-reported outcomes of an integrative pain management program implemented in a primary care safety net clinic: a quasi-experimental study. J Gen Intern Med 2019;34:1105-7. 
7. Hurstak E, Chao MT, Leonoudakis-Watts K, Pace J, Walcer B, Wismer B. Design, implementation, and evaluation of an integrative pain management program in a primary care safety-net clinic. J Altern Complement Med 2019;25:S78-S85.

8. Knight KR, Kushel M, Chang JS, et al. Opioid pharmacovigilance: a clinical-social history of the changes in opioid prescribing for patients with cooccurring chronic non-cancer pain and substance use. Soc Sci Med 2017;186:87-95.

9. Hirsh AT, Atchison JW, Berger JJ, et al. Patient satisfaction with treatment for chronic pain: predictors and relationship to compliance. Clin J Pain 2005;21:302-10.

10. Prakash B. Patient satisfaction. J Cutan Aesthet Surg 2010;3:151-5.

11. Gruß I, Firemark A, McMullen CK, Mayhew M, DeBar LL. Satisfaction with primary care providers and health care services among patients with chronic pain: a mixed-methods study. J Gen Intern Med 2020;35:190-7.

12. Sherwood G, Adams-McNeill J, Starck PL, Nieto B, Thompson CJ. Qualitative assessment of hospitalized patients' satisfaction with pain management. Res Nurs Health 2000;23:486-95.

13. Isenberg SR, Maragh-Bass AC, Ridgeway K, Beach MC, Knowlton AR. A qualitative exploration of chronic pain and opioid treatment among HIV patients with drug use disorders. J Opioid Manag 2017;13:5-16.

14. Dassieu L, Kaboré J-L, Choinière M, Arruda N, Roy É. Chronic pain management among people who use drugs: a health policy challenge in the context of the opioid crisis. Int J Drug Policy 2019;71:150-6.

15. Edlund MJ, Martin BC, Devries A, Fan M-Y, Braden JB, Sullivan MD. Trends in use of opioids for chronic non-cancer pain among individuals with mental health and substance use disorders: the TROUP study. Clin J Pain 2010;26:1-8.

16. Vijayaraghavan M, Penko J, Guzman D, Miaskowski C, Kushel MB. Primary care providers' views on chronic pain management among highrisk patients in safety net settings. Pain Med 2012;13:1141-8.

17. Guy GP, Zhang K, Bohm MK, et al. Vital signs: changes in opioid prescribing in the United States, 2006-2015. MMWR Morb Mortal Wkly Rep 2017;66:697-704.

18. Bohnert ASB, Guy GP, Losby JL. Opioid prescribing in the United States before and after the Centers for Disease Control and Prevention's 2016 opioid guideline. Ann Intern Med 2018;169: 367-75.

19. Chang JS, Kushel M, Miaskowski C, et al. Provider experiences with the identification, management, and treatment of co-occurring chronic non-cancer pain and substance use in the safety net. Subst Use Misuse 2017;52:251-5.

20. Busch SH, Meara E, Huskamp HA, Barry CL. Characteristics of adults with substance use disorders expected to be eligible for Medicaid under the ACA. PS 2013;64:520-6.

21. Laverdiere D, Pereyda M, Silva J, Tatar M. Changing course: the role of health plans in curbing the opioid epidemic. Oakland: California Health Care Foundation; 2016. p. 35. Available from: https://www.chcf.org/wp-content/uploads/2017/12/ PDF-ChangingHealthPlansOpioid.pdf.

22. American Medical Association. National roadmap on state-level efforts to end the opioid epidemic. Chicago: AMA; 2019. p. 27. Available from: https:// www.ama-assn.org/delivering-care/opioids/reversingopioid-epidemic.

23. Coffin PO, Rowe C, Gerbino N. Substance use trends in San Francisco through 2019. Gainesville, FL: National Drug Early Warning System (NDEWS) Coordinating Center; 2020. Available from: https:// www.csuhsf.org/substance-use-trends-san-francisco.

24. Dowell D, Haegerich TM, Chou R. CDC Guideline for Prescribing Opioids for Chronic Pain-United States, 2016. JAMA 2016;315:1624-45.

25. Denis CM, Morales KH, Wu Q, Metzger DS, Cheatle MD. Association between diagnoses of chronic noncancer pain, substance use disorder, and HIV-related outcomes in people living with HIV. J Acquir Immune Defic Syndr 2019;82 Suppl 2: S142-S147.

26. Piper BJ, Beals ML, Abess AT, et al. Chronic pain patients' perspectives of medical cannabis. Pain 2017;158:1373-9.

27. Tate CC, Ledbetter JN, Youssef CP. A two-question method for assessing gender categories in the social and medical sciences. J Sex Res 2013;50: 767-76.

28. Prins A, Ouimette P, Kimerling R, et al. The Primary Care PTSD Screen (PC-PTSD): development and operating characteristics. Prim Care Psych 2004;9:9-14.

29. Kroenke K, Strine TW, Spitzer RL, Williams JBW, Berry JT, Mokdad AH. The PHQ-8 as a measure of current depression in the general population. J Affect Disord 2009; 114:163-73.

30. Dhingra SS, Kroenke K, Zack MM, Strine TW, Balluz LS. PHQ-8 days: a measurement option for DSM-5 Major Depressive Disorder (MDD) severity. Popul Health Metr 2011;9:11.

31. Recklitis CJ, Blackmon JE, Chang G. Validity of the Brief Symptom Inventory-18 (BSI-18) for identifying depression and anxiety in young adult cancer survivors: comparison with a structured clinical diagnostic interview. Psychol Assess 2017;29:1189-200.

32. Kerper LF, Spies CD, Tillinger J, et al. Screening for depression, anxiety, and general psychological 
distress in pre-operative surgical patients: a psychometric analysis of the Patient Health Questionnaire 4 (PHQ-4). clinhp 2014;5-14.

33. Derogatis LR. Brief Symptom Inventory (BSI)-18: administration, scoring and procedures manual; 2001. Available from: http://www.pearsonclinical. com/psychology/products/100000638/brief-symptominventory-18-bsi-18.html.

34. Leung L. Pain catastrophizing: an updated review. Indian J Psychol Med 2012;34:204-17.

35. Osman A, Barrios FX, Gutierrez PM, Kopper BA, Merrifield T, Grittmann L. The Pain Catastrophizing Scale: further psychometric evaluation with adult samples. J Behav Med 2000;23:351-65.

36. Kortlever JTP, Janssen SJ, van Berckel MMG, Ring D, Vranceanu AM. What is the most useful questionnaire for measurement of coping strategies in response to nociception? Clin Orthop Relat Res 2015;473:3511-8.

37. Hansen L, Penko J, Guzman D, Bangsberg DR, Miaskowski C, Kushel MB. Aberrant behaviors with prescription opioids and problem drug use history in a community-based cohort of HIV-infected individuals. J Pain Symptom Manage 2011;42:893902.

38. Penko J, Mattson J, Miaskowski C, Kushel M. Do patients know they are on pain medication agreements? Results from a sample of high-risk patients on chronic opioid therapy. Pain Med 2012;13: 1174-80.

39. McIntyre MH, Kless A, Hein P, Field M, Tung JY, Team 23andMe Research. Validity of the cold pressor test and pain sensitivity questionnaire via online self-administration. PLoS One 2020;15: e0231697.

40. Modir JG, Wallace MS. Human experimental pain models 2: the cold pressor model. Methods Mol Biol 2010;617:165-8.

41. Wilkins CH, Roe CM, Morris JC. A brief clinical tool to assess physical function: the mini-physical performance test. Arch Gerontol Geriatr 2010;50: 96-100.

42. Morasco BJ, Lovejoy TI, Turk DC, Crain A, Hauser P, Dobscha SK. Biopsychosocial factors associated with pain in veterans with the hepatitis $\mathrm{C}$ virus. J Behav Med 2014;37:902-11.

43. Centers for Disease Control and Prevention. Calculating total daily dose of opioids for safer dosage; 2019. Available from: https://www.cdc.gov/ drugoverdose/pdf/prescribing/Guidelines_Factsheeta.pdf.

44. Nielsen S, Degenhardt L, Hoban B, Gisev N. A synthesis of oral morphine equivalents (OME) for opioid utilisation studies. Pharmacoepidemiol Drug Saf 2016;25:733-7.

45. Centers for Disease Control and Prevention. Pocket guide: tapering opioids for chronic pain.
Available from: https://www.cdc.gov/drugoverdose/ pdf/clinical_pocket_guide_tapering-a.pdf.

46. Kertesz SG. Outcomes after opioid dose reductions and stoppage: it's time to start counting. J Subst Abuse Treat 2019;103:64-5.

47. Kertesz SG, Manhapra A, Gordon AJ. Nonconsensual dose reduction mandates are not justified clinically or ethically: an analysis. J Law Med Ethics 2020; 48:259-67.

48. Frank JW, Lovejoy TI, Becker WC, et al. Patient Outcomes in Dose Reduction or Discontinuation of Long-Term Opioid Therapy. Ann Intern Med 2017;167:181-91.

49. McPherson S, Smith CL, Dobscha SK, et al. Changes in pain intensity after discontinuation of long-term opioid therapy for chronic noncancer pain. Pain 2018;159:2097-104.

50. Coffin PO, Rowe C, Oman N, et al. Illicit opioid use following changes in opioids prescribed for chronic non-cancer pain. PLoS ONE 2020;15: e0232538.

51. James JR, Scott JM, Klein JW, et al. Mortality after discontinuation of primary care-based chronic opioid therapy for pain: a retrospective cohort study. J Gen Intern Med 2019;34:2749-55.

52. Demidenko MI, Dobscha SK, Morasco BJ, Meath THA, Ilgen MA, Lovejoy TI. Suicidal ideation and suicidal self-directed violence following clinicianinitiated prescription opioid discontinuation among long-term opioid users. Gen Hosp Psychiatry 2017;47:29-35

53. Oliva EM, Bowe T, Manhapra A, et al. Associations between stopping prescriptions for opioids, length of opioid treatment, and overdose or suicide deaths in US veterans: observational evaluation. BMJ 2020;m283.

54. Mark TL, Parish W. Opioid medication discontinuation and risk of adverse opioid-related health care events. Subst Abuse 2019;103:58-63.

55. Fischer B, Jones $W$, Tyndall $M$, Kurdyak $P$. Correlations between opioid mortality increases related to illicit/synthetic opioids and reductions of medical opioid dispensing-exploratory analyses from Canada. BMC Public Health 2020;20:143.

56. Behar E, Bagnulo R, Knight K, Santos G-M, Coffin PO. "Chasing the pain relief, not the high": experiences managing pain after opioid reductions among patients with HIV and a history of substance use. PLoS One 2020;15:e0230408.

57. Kroenke K, Alford DP, Argoff C, et al. Challenges with implementing the Centers for Disease Control and Prevention opioid guideline: a consensus panel report. Pain Med 2019;20:724-35.

58. Glowacki D. Effective pain management and improvements in patients' outcomes and satisfaction. Crit Care Nurse 2015;35:33-41.

59. Hassan AN, Le Foll B, Imtiaz S, Rehm J. The effect of post-traumatic stress disorder on the risk of 
developing prescription opioid use disorder: results from the National Epidemiologic Survey on Alcohol and Related Conditions III. Drug Alcohol Depend 2017;179:260-6.

60. Park TW, Lin LA, Hosanagar A, Kogowski A, Paige K, Bohnert ASB. Understanding risk factors for opioid overdose in clinical populations to inform treatment and policy. J Addict Med 2016;10:369-81.

61. Cody GR, Wang B, Link AR, Sherman SE. Characteristics of urban inpatient smokers with and without chronic pain: foundations for targeted cessation programs. Subst Use Misuse 2019;54:1138-45.

62. Zvolensky MJ, McMillan K, Gonzalez A, Asmundson GJG. Chronic pain and cigarette smoking and nicotine dependence among a representative sample of adults. Nicotine Tob Res 2009;11:1407-14.

63. Goesling J, Brummett CM, Meraj TS, Moser SE, Hassett AL, Ditre JW. Associations between pain, current tobacco smoking, depression, and fibromyalgia status among treatment-seeking chronic pain patients. Pain Med 2015;16:1433-42.

64. Bonnie RJ, Stratton K, Kwan LY. The effects of tobacco use on health; 2015. Available from: https:/www.ncbi.nlm.nih.gov/books/NBK310413/.

65. Cunningham CO. Opioids and HIV infection: from pain management to addiction treatment. Top Antivir Med 2018;25:143-6.

66. Edelman EJ, Gordon K, Becker WC, et al. Receipt of opioid analgesics by $\mathrm{HIV}$-infected and uninfected patients. J Gen Intern Med 2013;28:82-90.

67. Horberg MA, Hurley LB, Towner WJ, et al. Determination of optimized multidisciplinary care team for maximal antiretroviral therapy adherence. J Acquir Immune Defic Syndr 2012;60:183-90.

68. Zaller N, Gillani FS, Rich JD. A model of integrated primary care for HIV-positive patients with underlying substance use and mental illness. AIDS Care 2007;19:1128-33.

69. Merlin JS, Long D, Becker WC, et al. Brief report: the association of chronic pain and long-term opioid therapy with HIV treatment outcomes. J Acquir Immune Defic Syndr 2018;79:77-82.

70. Starrels JL, Peyser D, Haughton L, et al. When human immunodeficiency virus (HIV) treatment goals conflict with guideline-based opioid prescribing: a qualitative study of HIV treatment providers. Subst Abuse 2016;37:148-53.

71. Cuca YP, Shumway M, Machtinger EL, et al. The association of trauma with the physical, behavioral, and social health of women living with HIV: pathways to guide trauma-informed health care interventions. Womens Health Issues 2019;29:376-84.

72. Sohler NL, Starrels JL, Khalid L, et al. Cannabis use is associated with lower odds of prescription opioid analgesic use among HIV-infected individuals with chronic pain. Subst Use Misuse 2018;53: 1602-7.

73. Merlin JS, Long D, Becker WC, et al. Marijuana use is not associated with changes in opioid prescriptions or pain severity among people living with $\mathrm{HIV}$ and chronic pain. J Acquir Immune Defic Syndr 2019;81:231-7.

74. Merlin JS, Samet JH, Cheng DM, et al. Marijuana use and its associations with pain, opioid dose, and HIV viral suppression among persons living with HIV on chronic opioid therapy. J Acquir Immune Defic Syndr 2019;82:195-201.

75. Romero-Sandoval EA, Kolano AL, AlvaradoVázquez PA. Cannabis and cannabinoids for chronic pain. Curr Rheumatol Rep 2017;19:67.

76. Ishida JH, Wong PO, Cohen BE, Vali M, Steigerwald S, Keyhani S. Substitution of marijuana for opioids in a national survey of US adults. PLoS ONE 2019;14:e0222577.

77. Campbell G, Hall WD, Peacock A, et al. Effect of cannabis use in people with chronic non-cancer pain prescribed opioids: findings from a 4-year prospective cohort study. Lancet Public Health 2018;3: e341-e350.

78. Cooke AC, Knight KR, Miaskowski C. Patients' and clinicians' perspectives of co-use of cannabis and opioids for chronic non-cancer pain management in primary care. Int J Drug Policy 2019; 63:23-8

79. Deshpande A, Mailis-Gagnon A, Zoheiry N, Lakha SF. Efficacy and adverse effects of medical marijuana for chronic noncancer pain: systematic review of randomized controlled trials. Can Fam Physician 2015;61:e372-e381. 
Appendix 1. List of Detectable Substances on Urine Drug Screen

Amphetamines

Barbiturates

Benzodiazepines

Buprenorphine

Cocaine (detected as benzoylecgonine)

Methadone (detected as methadone and 2-ethylidene-1,5dimethyl-3,3-diphenylpyrrolidine [EDDP])

Methamphetamine

Opioids

Oxycodone

Phencyclidine (PCP)

Tetrahydrocannabinol (THC)

\section{Appendix 2. Data Analysis Supplement}

In the multivariable ordinal regression model assessing factors associated with pain treatment satisfaction, we included a priori defined independent variables (age and gender) and predictors from bivariate analysis with a $P$ value $<0.05$. When there were multiple variables with $P<.05$ in the bivariate analysis that were considered to measure a highly similar characteristic, we selected the variable thought most likely to influence pain treatment satisfaction. For example, we chose to include average pain score in the past 3 months in the multivariable analysis, rather than current pain, due to this measure having a larger relevance in clinical practice. We also considered taking no opioids and taking no medications for pain treatment to be highly similar with having opioids discontinued in the past year, as all individuals in the study had been on recent opioid therapy. Among these 3 measures, we included only opioid discontinuations in the final model, as it had the strongest association. Although reporting being on hydromorphone therapy and being positive for buprenorphine on the urine drug screen were also significantly associated with satisfaction in the bivariate analysis, we did not include these variables in the final model due to small sample sizes. We validated our multivariable ordinal logistic regression model by running the Brant test command, validating the proportional odds assumption, and running the linktest command, to detect any specification errors. The $P$ value for the Brant test was 0.623 , suggesting no violation of the proportional odds assumption. The $P$ value for the _hatsq variable on the linktest command was 0.914 , meaning no specification error was detected. We also checked for multicollinearity in the final model using the VIF command, and all VIF values were less than 10 , suggesting lack of collinearity between variables in the final model. 